\title{
Isolation of Sulforaphene and Sulforaphane, The Novel Anticancer Reagent, from Raphanus sativus
}

\section{Bing Lan L* \\ Head of Department at Chyaoyang University of Technology, Taiwan}

*Corresponding author: Bing-Lan Liu, Head of Department at Chyaoyang University of Technology, 168 Jifeng E. Rd, Wufeng District, Taichung, 41349, Taiwan, Tel: +886-4-23323000; E-mail: binglan@cyut.edu.tw

\section{Research Article}

Volume 1 Issue 2

Received Date: October 16, 2017

Published Date: November 10, 2017

\section{Abstract}

Raphanussativus (radish), a common cruciferous vegetable has been attributed to possess a number of pharmacological and therapeutic properties. Sulforaphene (SFE), one of bioactive constituents in radish, is an isothiocyanate that have a strong antioxidant compounds generally be found in Brassicaceae. SFE is a hydrolyzate form glucosinolates by myrosinase. An analytical method, utilizing HPLC and LC /MS, which allows to determine its absolute content in different parts of radish. In this study, the radish was pretreated and the contents of various parts were confirmed by HPLC. The highest content of SFE $(48.17 \pm 1.11 \mu \mathrm{g} / \mathrm{g})$ was found in the radish leaves, followed by the radish peeled root and the lowest is peel $(0.48 \pm 0.05 \mu \mathrm{g} / \mathrm{g})$. The SFE and its analogues, sulforaphane (SFN), could be partially purified by liquid-liquid extraction and silica gel column chromatography. Since the SFE and SFN can't not be directly identified by HPLC, two components were further confirmed by LC/MS after quantification. This is the first report regarding to the identification of these two constituents in the R.sativus extract, simultaneously. The unique functional groups for SFE/SFN were also confirmed by FTIR in radish sample.

Keywords: Sulforaphane; Glucosinolates; Radish; Spectrometry; Purification

\section{Introduction}

Brassicaceae and related plant families are rich in secondary plant metabolites including over 120 glucosinolates and various isothiocyanate [1-4]. A characteristic of cruciferous plants is the presence of thioglucosides, a class of secondary metabolite commonly known as glucosinolates, which on contact with myrosinasea potential role for the myrosinaseglucosinolate system in the cruciferous. Such system in turn is involved in a range of biological activities, by generating isothiocyanate, which act as synergists for the alarm pheromone to warning other members of a developing colony [6,7]. Several studies have revealed that various bioactive functions appear to be related to high amounts of glucosinolates and myrosinase in cruciferous vegetables. Among bioactive compounds in radish sprouts, sulforaphene (SFE: 4-methylsulfinyl-3butenylisothiocyanate) (Figure 1), an important isothiocyanatederived from glucoraphenin (GRE: 4methylsulfinyl-3-butenylglucosinolate), is strongly associated with anticancer activity [8]. Raphanussativus (radish) is a root vegetable of the Brassicaceae family, commonly seen as a small-rooted, short-season vegetable, normally consumed in salads [9]. Radish is widely grown all over the world and has been used for long history. Different parts of radish including roots, seeds and leaves are used for medicinal purposes [10]. $R$. sativus has been used ethnically as a laxative, stimulant, digestive aid, appetizer and in the treatment of stomach disorders [11]. The main constituents of radish are 4-(methylthio)-3-butenyl isothiocyanate, allyl isothiocayanate, benzyl isothiocyanate and phenethylisothiocyanate. It also contains flavonoids such as kaempherol glycosides, peroxidases and antioxidants [12-15]. The previous studies reported that $R$. sativusextract showed antimicrobial, antimutagenic and anticarcinogenic effects [10,16-17]. 
Sulforaphane (SFN: 1-isothiocyanato-4methylsulfinylbutane)(Figure 1), compound-based drug derived from dietary isothiocyanates which has previously been shown to possess potent anti-tumor and chemo preventive effects against several types of cancer, was found in broccoli (Brassica oleracea L. var. italica) [3]. The anti-cancer mechanism of SFN and SFE could be attributed to the following steps: (a) blocking the initiation state via inhibiting Phase I enzymes to convert procarcinogens to proximate or ultimate carcinogens; and (b) inducing Phase II enzymes that detoxify carcinogens and facilitate their excretion from the body [18-19]. A further protective effect associated with oxidative stress was revealed in experimental models [20]. SFE, with an additional double bond to SFN, has been reported to reduce cancer cell proliferation in a dose-dependent manner and induce apoptosis in many cancer cells [21-23]. Both SFN and SFE are bioactive constituents in Thai rat-tailed radish (R.sativus L. var.caudatusAlef, RS or Pak Khi Hood), contributing to an apoptosis induction effect against the HCT116colon cancer cell line [24].

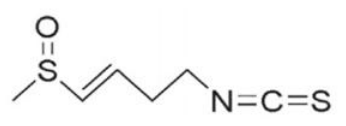

Sulforaphene

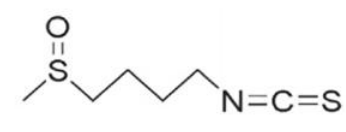

\section{Sulforaphane}

Figure 1: Chemical structures of sulforaphene (SFE) and sulforaphane (SFN).

Many researchers have focused on finding isothiocyanate (including SFE) in cruciferous crops, such as: broccoli, cabbage, cauliflower, as a potential chemo preventive source but rarely in R.sativus due to its relative low quantity as well as structural similarities between SFE and SFN. In the present study, we attempted to isolate the SFE from different parts of R.sativus and to identify SFE using a mass spectrometry.

\section{Materials and Methods}

\section{Materials and Reagents}

Raphanussativus was purchased from local market. Sulforaphane, sulforaphene, anhydrous $\mathrm{Na}_{2} \mathrm{SO}_{4}$, and silica gel were purchased from Sigma-Aldrich (St. Louis, MO). All other chemicals were of analytical grade, unless otherwise noted.

\section{Extraction and Isolation of Sulforaphene}

The radishes purchased from local market were cleaned and divide into three parts: stems, root, and the peeled root. Each part sample (100 g) was homogenized after adding the deionized water $(1: 3$, w/v) using blender for three hours $\left(37^{\circ} \mathrm{C}\right)$.Subsequently, the residue was filtered off by suction and centrifuged with the high-speed centrifuge to separate the smaller particles at 8,000 rpm for $15 \mathrm{~min}$. The partial moisture was removed by the decompression concentrator $\left(37^{\circ} \mathrm{C}\right.$, 20 mbar). Then, remove the precipitate by using the high-speed centrifuge at 8,000 rpm for $10 \mathrm{~min}$.

The liquid-liquid methods were be slightly modified in the research according to the reference described previously [25,26]. For liquid-liquid extraction, theme thylene chloride $(1: 1)$ was added to the supernatant (repeat 2 times). Take the organic layer, the anhydrous $\mathrm{Na}_{2} \mathrm{SO}_{4}$ was added to remove the moisture. The Rotary evaporator (R-210Buchi, Flawil, Switzerland) was used to decant the organic solvent. The crude extract was redissolved by methanol while the HPLC was employed for the quantitative determination of SFN.

The dry crude extract was redissolved in ethyl acetate (EA) and subjected to further chromatographic purification (Silica gel 60, Merck, 0.015-0.040 mm). Methylene chloride extracted sample was impregnated onto the silica gel, and loaded onto the column. The column $(30 \mathrm{~cm} \times 2.5 \mathrm{~cm})$ was equilibrated with $\mathrm{EA} / \mathrm{MeOH}$ (1:1) and eluted regularly as the polarity of the eluent increased. SFE/SFN was eluted with $100 \%$ EA, finally so thiocyanates were washed out with $100 \% \mathrm{MeOH}$. The solvents from the eluates were evaporated in vacuo. The final product was redissolved with $\mathrm{MeOH}$ prior to HPLC analysis. The whole purification process was depicted in Figure 2.

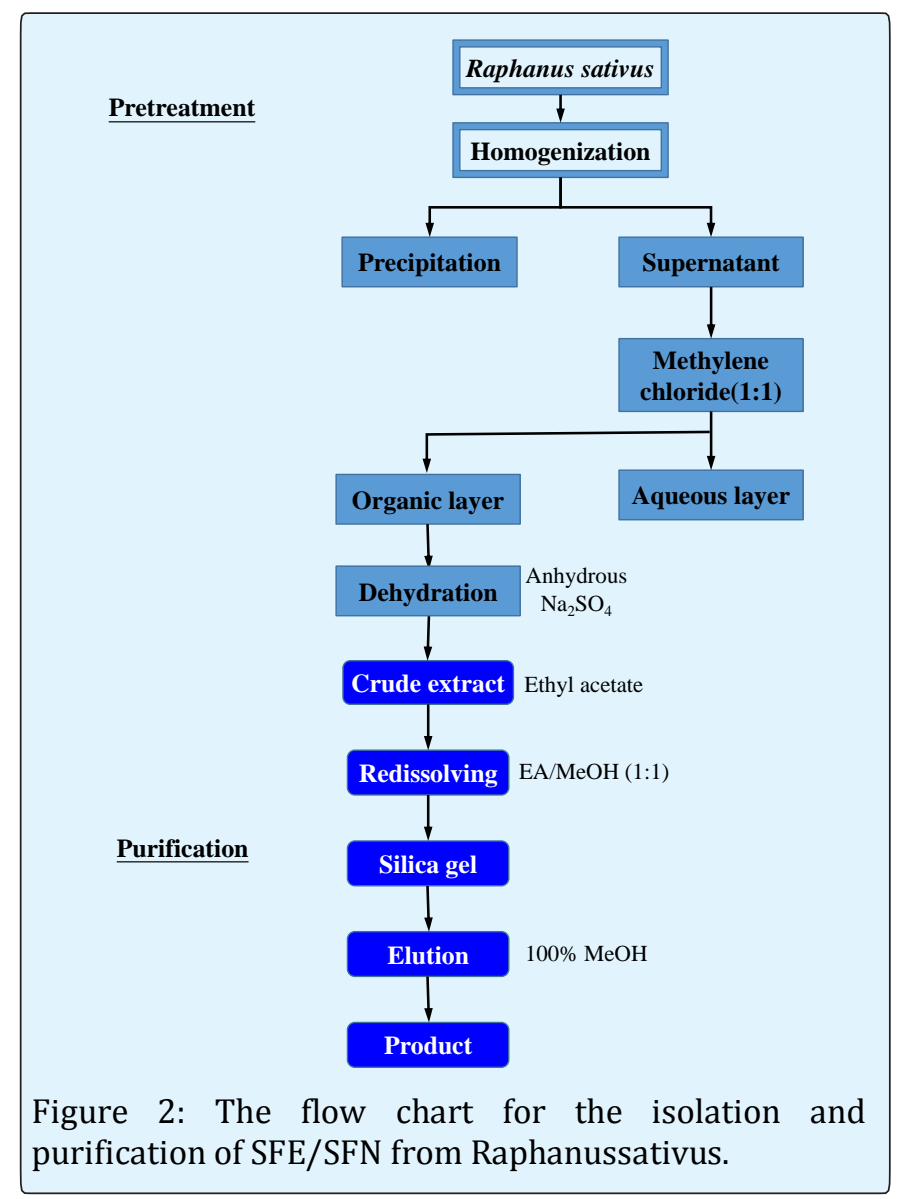




\section{Bioequivalence \& Bioavailability International Journal}

\section{HPLC Analysis}

The SFE was determined on an analytical HPLC system consisted of a Hitachi (Tokyo, Japan) Primaide 1410 UV-Vis detector, Primaide 1110 pump, Hitachi D2000 Elite Software and a EC 250/4.6 nucleodur column (4.6×250 mm, $5 \mu \mathrm{m}$, Germany).Mobile phase composed of solvents $\mathrm{A}$ and $\mathrm{B}$ were water and acetonitrile, respectively. The solvent gradient was programmed as follows: from 20 to $60 \%$ A for $15 \mathrm{~min}$ at a flow rate of 1 $\mathrm{mL} / \mathrm{min}$. A $5 \mu \mathrm{L}$ sample was injected and detected at $254 \mathrm{~nm}$. The SFE standards ranging from 1.56 to 25.00 $\mu \mathrm{g} / \mathrm{mL}$ were used to construct a standard calibration curve. Samples were filtered through a $0.22-\mu \mathrm{m}$ membrane disc before HPLC analysis.

\section{Analysis of SFE by ATR/FT-IRS spectroscopy}

The sample was dissolved in $\mathrm{Me} \mathrm{OH}$ and the Fourier transformed infrared spectroscopy (FTIR) was performed on a Perkin-Elmer Spectrum 10 $10^{\mathrm{TM}}$ FTIR Spectrophotometer (Perkin-Elmer, Waltham, MA)equipped with a liquid nitrogen cooled mercury/cadmium telluride detector and a $45^{\circ}$ horizontal zinc selenide (ZnSe) crystal as an ATR accessory(Pike Technologies, Madison, WI). An open beam background spectrum was collected through the clean ZnSe crystal after rinsing it with chloroform. For the collection of sample spectra, $0.5 \mathrm{~mL}$ of sample solution in chloroform was layered onto the crystal, and the solvent was allowed to dry before collecting the inter ferograms of the sample film formed on the ATR crystal. Each sample interferogram was ratioed against the background interferogram, and the resulting transmission spectrum was transformed. The spectra were scanned in the range $4000 \mathrm{~cm}^{-1}$ to $800 \mathrm{~cm}^{-1}$ by collecting and averaging 16 spectra.

\section{LC/MS Analysis}

The LC/MS analysis was performed on a Agilent 1100 series LC system (Waldbronn, Germany) coupled with a model LCQ advantage mass spectrometer system (ThermoFinnigan, San Jose, CA) equipped with ESI ion source. The sheath gas flow rate was $20 \mathrm{arb}$, spray voltage was $4.5 \mathrm{kV}$, capillary temperature and capillary voltage was $200^{\circ} \mathrm{C}$ and $10.0 \mathrm{~V}$. A $10 \mu \mathrm{L}$ sample was injected and detected positive ions mode for $3 \mathrm{~min}$.

\section{Results and Discussion}

\section{Analysis of Extracts and Standard by HPLC}

Gradient elution of HPLC chromatograms for standard SFE and SFN were presented in Figure 3(A) The SFE and SFN had retention times of $9.4 \sim 9.5$ mins on the nucleodur column. As can be seen, the SFE and SFN nearly overlap in the HPLC chromatograms due to the structure difference only in one $\mathrm{C}-\mathrm{C}$ and $\mathrm{C}=\mathrm{C}$, respectively. Yet, the HPLC bearing the high theoretical plates (resolution), the SFE and SFN are still not easy to separate under normal HPLC conditions (Figure 3A). A number of previous studies had tried to detect more than one major compound in the crude extract mixture. The HPLC elucidation profile of R.sativus crude extract in the previous reports suggested the peaks for SFE and SFN coexisted [24,25].

The calibration curve showed the linearity of the detector over the tested range $(1.52 \sim 25 \mathrm{mg} / \mathrm{mL})$ Linear regression analysis of the peak area responses (y) versus the theoretical concentration $(x)$ gave the following equation: $\mathrm{y}=1650.61+517.55 \mathrm{x}, r^{2}=0.9912$ (Figure 3B). The correlation coefficient demonstrated linearity of the method over the concentration range. Average standard errors for the peak areas of replicate injections were smaller than 5\%, showing a good repeatability of the calibration curve. The system precision was determined by chromatography five injections of the standard solution and calculating the relative standard deviation (R.S.D.) of the peak area responses. The method was used to quantitatively determine SFE in each part of radish. As summarized in (Table 1), the highest content of SFE was found in the leaves, followed by the peeled root and the last one is peel. The high desired compound contents indicate that the radish leaves is good raw materials for preparing SFE. The lowest content of isothiocyanate (SFE/SFN) was found in peel, may beyond our expectation while its majority in daily used. This may partially due to the reduction of myrosinase activity in Rsativus during the summer. The previous researches have also shown that the formation of a bioactive compound, SFE in radish roots, was influenced by temperature [28]. The lower in the temperature, the greatest formation of SFE was observed in which the myrosinase activity was also maintained in the highest level. Subsequently, the SFE productions were correlated with myrosinase activity in radish roots.

\begin{tabular}{|c|c|}
\hline Radish sample & Content of SFE $(\boldsymbol{\mu g} / \mathbf{g})$ \\
\hline Leaves & $48.17 \pm 1.11$ \\
\hline Peeled Root & $18.93 \pm 1.62$ \\
\hline Peel & $0.48 \pm 0.05$ \\
\hline
\end{tabular}

Table 1: Quantitative determination of SFE in the different parts of R.sativus.

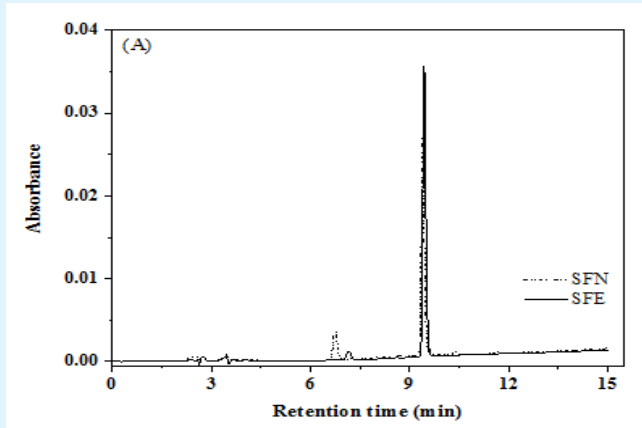

Figure 3(A): The HPLC chromatograms of SFE and SFN standards (100 ppm) 


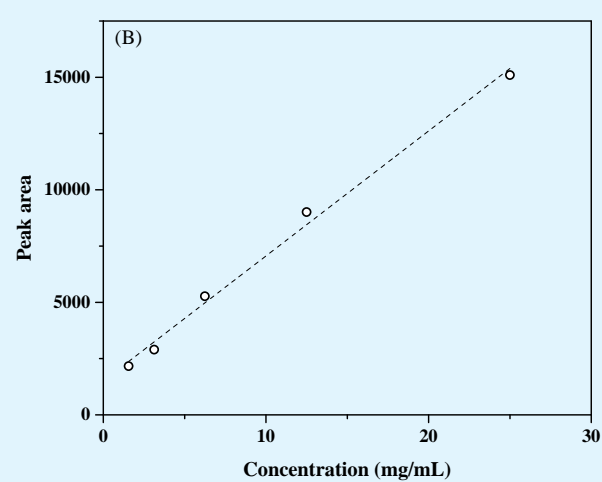

Figure 3(B): The standard calibration curve of SFE.

\section{Isolation and Purification of SFE}

The SFE/SFN in the different parts of R. sativus was further purified by Methylene chloride extraction and column chromatography. The SFE/SFN was monitored during the purification steps by TLC (data not shown) or HPLC. The HPLC chromatogram of the sample before and after silica gel purification was given in Figure 4 Apparently; most impurities were removed during the isolation and purification steps (c.f. Figure 2). The SFE/SFN still retain at 9.4 mins after silica gel chromatography, while the other constituents were disappeared The $R$. sativusextract have been reached almost $80 \%$ purity based on the peak area after liquidliquid extraction and silica gel chromatography. Both SFE/SFN could be simply isolated from the R. sativus crude sample through liquid-liquid extraction and column chromatography. This sample was provided for further FTIR and LC/MS analysis.

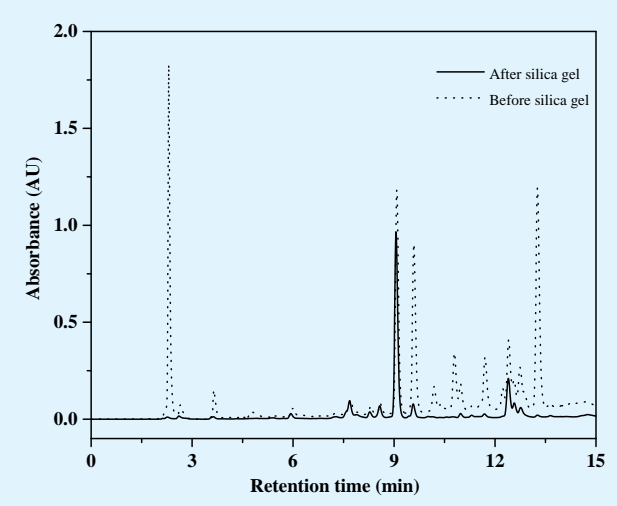

Figure 4: The HPLC chromatograms of R.sativus sample before and after silica gel column chromatography

\section{FTIR Analysis}

FT-IR measurements of the SFE/SFN standard and $R$. sativus purified sample showed a similar absorption profile (Figure 5). A band at $2915 \mathrm{~cm}^{-1}$ which was due to the $-\mathrm{C}-\mathrm{H}$ stretching band of backbone in SFE/SFN (Figure 5 top). The unique stretching of $-\mathrm{N}=\mathrm{C}=\mathrm{O}$,
$-\mathrm{N}=\mathrm{C}=\mathrm{S},-\mathrm{N}=\mathrm{C}=\mathrm{N}-,-\mathrm{N}_{3}, \mathrm{C}=\mathrm{C}=0$ groups in isothiocyanate were in the range of $2100 \sim 2270 \mathrm{~cm}^{-1}$ [24]. Thus, the presence of SFN/SFE could be characterized by the absorption and centered at $2101.98 \mathrm{~cm}^{-1}$.In addition, an alkenes $\mathrm{C}=\mathrm{C}$ stretch governed an absorption band between $1690 \sim 1630 \mathrm{~cm}^{-1}$ was found in the case of SFE but not in the SFN (Figure 5 top). Alternatively, the single C-C bonds absorb around $1200 \mathrm{~cm}^{-1}$ was found in SFN. Both functional groups were appeared in the R.sativus purified sample (Figure 5 bottom). And absorption band appeared at $1450 \mathrm{~cm}^{-1}$ corresponding to another function group $(-S=0)$ in plane bending of backbone of SFE/SFN.

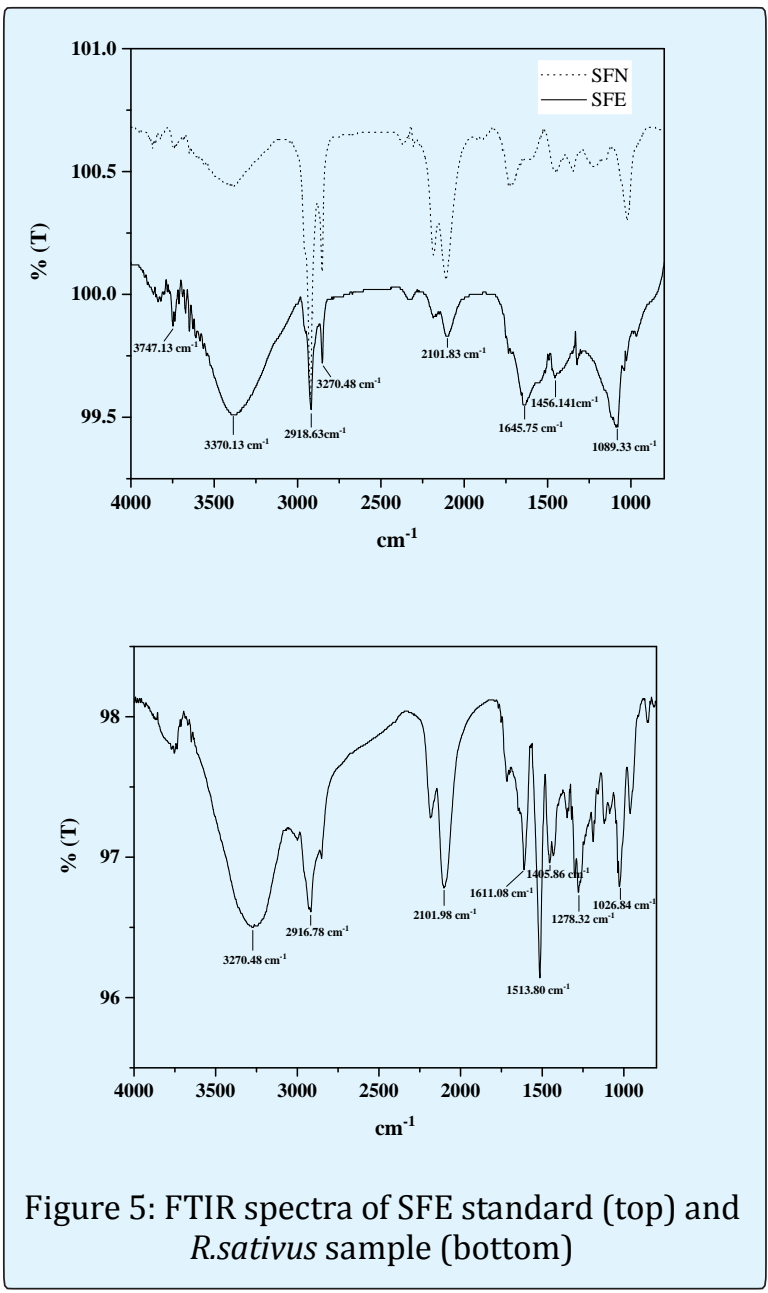

\section{LC/MS Analysis}

HPLC associated with electro spray soft ionization mass spectroscopy (LC-ESI-MS) system was used to identify the molecular weight of purified sample from $R$. sativus. Samples were conveniently detected by negative ionization electro spray LC/MS. Fig. 6 shows the commercial standards for SFN/SFE and purified sample. The stand significant ions occurred at $\mathrm{m} / \mathrm{z}$ $176\left(175+[\mathrm{H}]^{+}\right)$and $\mathrm{m} / \mathrm{z} 178\left(177+[\mathrm{H}]^{+}\right)$of SFE and SFN, respectively (Figure 6 top panel and middle panel). Accordingly, an alkyl group and alkenyl group could be distinguished using mass spectroscopy which in turn 
could not be separated from purified sample using HPLC. In fact, the LC-MS/MS method has been developed to determine the SFN in rat plasma, and was used to determine the absolute bioavailability of dietary doses of SFN following oral and intravenous administration [29]. Remarkable efficiency could be observed while the purified R.sativus was introduced into the LC/MS (Figure 6 bottom panel), two components have isolate peak. The mass spectrum revealed that the SFN also presented in R.sativus sample with relative low content. About 1 in 5 for SFN compared to SFE in the sample.

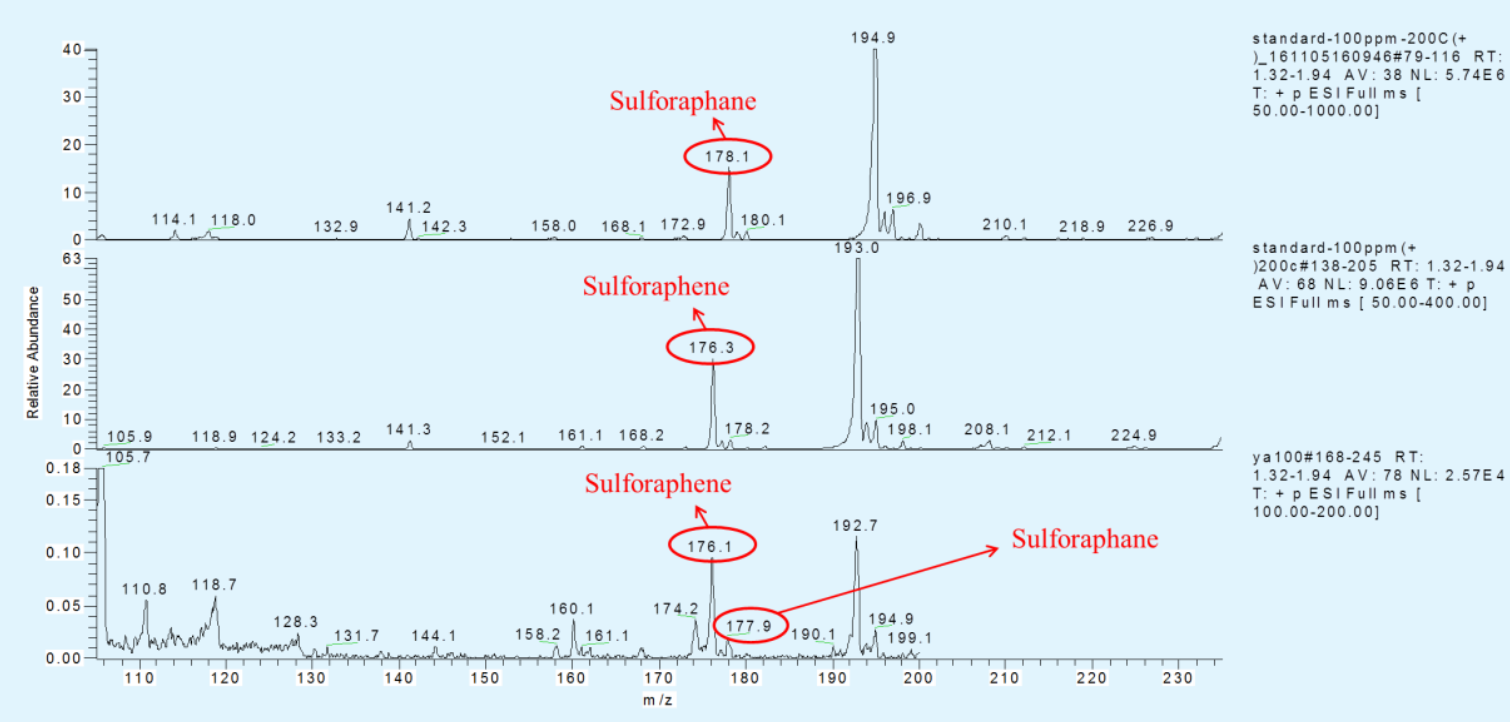

Figure 6: LC- ESI-MS base peak chromatograms in negative ion mode for SFE standard (top panel), SFN (middle panel) and purified sample of R.sativus (bottom panel).

\section{Conclusion}

In this study, the hydrolysis yield of glucosinolates product, SFE, was investigated from different parts of radish. Purification of SFE/SFN could be isolated using liquid-liquid extraction followed by solid phase extraction. Furthermore, the gradient elution was carried out for further polishing the purity of sulforaphene. Subsequently, determination of SFE was accomplished by RP-HPLC. The highest content of SFE was found to be $48.17 \pm 1.11 \mu \mathrm{g} / \mathrm{g}$ in the radish leaves. Coexistence of SFE/SFN was revealed by LC/MS. Thus, although the predominant type of isothiocyanate is SFE in $R$. sativus extract, the minor SFN in the sample still observed. This is the first report using LC/MS to identified the coexistence of these two compounds. Furthermore, the -SO was confirmed by the appearance of stretching frequency at $1350 \sim 1450 \mathrm{~cm}^{-1}$ in FTIR spectra. FTIR absorption bands at $2100 \sim 2270$ and $1780 \sim 1620 \mathrm{~cm}^{-1}$ due to $-\mathrm{N}=\mathrm{C}=\mathrm{S}$ and carbonyl group of acyl isothiocyanate in FTIR spectra confirmed SFE/SFN presence. At the same time, the SFE and its derivate, SFN, were found to be coexist in $R$. sativus extract as confirmed by LC/MS. The major component is SFE whereas the SFN presented in relative low content. Furthermore, the contents of sulforaphene in different parts of radish has been quantified. The further studies in the antibacterial test and other bioactive properties are under construction.

\section{References}

1. De Nicola GR, Bagatta M, Pagnotta E, Angelino D, Gennari L, et al. (2013) Comparison of bioactive phytochemical content and release of isothiocyanate in selected brassica sprouts. Food Chem 141(1): 297-303.

2. Li L, Lee W, Lee WJ, Auh JH, Kim SS, et al. (2010) Extraction of allyl isothiocyanate from Wasabi (Wasabia japonica Matsum) using supercritical carbon dioxide. Food Science and Biotechnology 19(2): 405-410.

3. Nakamura Y (2009) Chemoprevention by isothiocyanates: Molecular basis of apoptosis induction molecular basis of apoptosis induction. In: Yoshikawa T (Ed.). Chemoprevention and cancer: Food factors for health promotion 61: 170-181.

4. Oerlemans K, Barrett DM, SuadesCB, Verkerk R, Dekker M (2006) Thermal degradation of glucosinolates in red cabbage. Food Chemistry 95(1): 19-29.

5. Dawson GW, Griffiths DC, Pickett JA, WadhamsLJ, Woodcock CM (1987) Plant derived synergists ofalarm pheromone of turnip aphid Lipaphis (Hyadaphis) erysimi (Homoptera, Aphididae). Journal of Chemical Ecology 13(7): 1663-1671. 
6. Bridges M, Jones AME, Bones AM, Hodgson C, Cole R, et al. (2002) Spatial organization of the glucosinolate-myrosinase system in brassica specialist aphids is similar to that of the host plant. Proc Biol Sci. 269 (1487): 187-191.

7. Daxenbichler ME, VanEtten CH, Spencer GF (1977) Glucosinolates and derived products in cruciferous vegetables Identification of organic nitriles from cabbage. J. Agric. Food Chem 25(1): 121-124.

8. Li R, Song D, Vriesekoop F, Cheng Li, Yuan Q, et al. (2017) Glucoraphenin, sulforaphene, and anti proliferative capacity of radish sprouts in germinating and thermal processes. European Food Research and Technology 243(4): 547-554.

9. Curtis IS (2003) The noble radish: past, present and future. Trends in Plant Sciences 8(7): 305-307.

10. Lee SW, Yang KM, Kim JK, Nam BH, Lee CM, et al. (2012) Effects of white radish (Raphanussativus) enzyme extract on hepatotoxicity. Toxicol Res 28(3): 165-172.

11. Ahn MJ, Moon JW, Park CN, Bang HJ, Kim GO, et al. (2016) Chungpihongsim radish (Raphanussativus $L$. cv. Chungpihongsim) ameliorates ethanol-induced gastric injury in rats. Oriental Pharmacy and Experimental Medicine 16(1): 37-43.

12. Wang L, Burhenne K, Kristensen BK, Rasmussen SK (2004)Purification and cloning of a Chinese red radish peroxidase that metabolisepelargonidin and forms a gene family in Brassicaceae. Gene 343(2): 323-335.

13. Suh SJ, Moon SK, Kim CH (2006) Raphanussativus and its isothiocyanates inhibit vascular smooth muscle cells proliferation and induce $G(1)$ cell cycle arrest. International Immunopharmacology 6(5): 854-861.

14. Hashimoto T, Ueda Y, Oi N, Sakakibara H, Piao C, et al. (2006) Effects of combined administration of Quercetin, Rutin and extract of white radish sprout rich in Kaemferol glycosides on the metabolism in rats. Bioscience, Biotechnology, and Biochemistry 70(1): 279-281.

15. Lugasi A, Dwoeschák E, Blázovics A, Kéry Á (1998) Antioxidant and free radical scavenging properties of squeezed juice from black radish (Raphanussativus L. varniger) root. Phytotherapy Research 12(7): 502-506.

16. Hecht SS, Kenney PM, Wang M, Trushin N, Upadhyaya P (2000) Effects of phenethylisothiocyanate and benzyl isothiocyanate, individually and in combination, on lung tumorigenesis induced in $\mathrm{A} / \mathrm{J}$ mice by benzo[a]pyrene and 4-(methylnitrosamino)- 1-(3pyridyl)-1-butanone. Cancer Letters 150(1): 49-56.

17. Hashem FA, Saleh MM (1999) Antimicrobial components of some cruciferae plants (DiplotaxisharraForsk. and Erucariamicrocarpa Boiss.). Phyto therapy Research 13(4): 329-332.

18. Devi JR, Thangam EB (2012) Mechanisms of anticancer activity of sulforaphane from Brassica oleracea in HEp-2 human epithelial carcinoma cell line. Asian Pac J Cancer Prev 13(5): 2095-2100.

19. Yang M, Wang HY, Zhou M, Liu WL, Kuang PQ, et al. (2016) The natural compound sulforaphene, as a novel anticancer reagent, targeting PI3K-AKT signaling pathway in lung cancer. Oncotarget $7(47)$ : 76656-76666.

20. Guerrero-Beltrán CE, Mukhopadhyay $P$, Horváth $B$, Rajesh M, Tapia E, et al. (2012) Sulforaphane, a natural constituent of broccoli, prevents cell death and inflammation in nephropathy. J Nutr Biochem 23(5): 494-500.

21. Barillari J, Iori R, Papi A, Orlandi M, Bartolini G, et al. (2008) Kaiware Daikon (Raphanussativus L.) extract: A naturally multipotent chemo preventive agent. Journal of Agricultural and Food Chemistry 56(17): 7823-7830.

22. Papi A, Orlandi M, Bartolini G, Barillari J, Iori R, et al. (2008) Cytotoxic and antioxidant activity of 4methylthio-3-butenyl isothiocyanate from Raphanussativus L. (Kaiware Daikon) sprouts. Journal of Agriculture and Food Chemistry 56(3): 875-883.

23. Nastruzzi C, Cortesi R, Esposito E, Menegatti E, Leoni O, et al. (2000) In vitro Antiproliferative activity of isothiocyanates and nitriles generated by myrosinase-mediated hydrolysis of glucosinolates from seeds of cruciferous vegetables. Journal of Agricultural and Food 48(8): 3572-3575.

24. Pocasap P, Weerapreeyakul N, Barusrux S (2013) Cancer preventive effect of Thai rat-tailed radish (Raphanussativus L. var. caudatus Alef). Journal of Functional Foods 5(3): 1372-1381.

25. Liang H, Li C, Yuan Q, Vriesekoop F (2007) Separation and purification of sulforaphane from broccoli seeds by solid phase extraction and preparative high-performance liquid chromatography. Journal of Agricultural and Food Chemistry 55(20): 8047-8053.

26. Lim S, Lee J, Kim JK (2009) Analysis of isothiocyanates in newly generated vegetables: 


\section{Bioequivalence \& Bioavailability International Journal}

baemuchae (x Brassicoraphanus) as affected by growth. International Journal of Food Science and Technology 44(7): 1401-1407.

27. Sangthong S, Weerapreeyakul N (2016) Simultaneous quantification of sulforaphene and sulforaphane by reverse phase HPLC and their content in Raphanussativus L. var. caudatus Alef extracts. Food Chemistry 201: 139-144.

28. Lim SY, Lee EJ, Kim JK (2015) Decreased sulforaphene concentration and reduced myrosinase activity of radish (Raphanussativus L.) root during cold storage. Postharvest Biology and Technology 100: 219-225.

29. Hanlon N, Coldham N, Gielbert A, Kuhnert N, Sauer MJ, et al. (2008) Absolute bioavailability and dosedependent pharmacokinetic behaviour of dietary doses of the chemo preventive isothiocyanate sulforaphane in rat. British Journal of Nutrition 99(3): 559-564. 